\title{
A Conditional Economic Incentive Fails to Improve Linkage to Care and Antiretroviral Therapy Initiation Among HIV-Positive Adults in Cape Town, South Africa
}

\author{
Brendan Maughan-Brown, PhD, ${ }^{1}$ Philip Smith, MSocSci, Caroline Kuo, DPhil, ${ }^{3,4}$ Abigail Harrison, PhD, \\ Mark N. Lurie, PhD, Linda-Gail Bekker, $\mathrm{PhD}^{2}$, and Omar Galárraga, $\mathrm{PhD}^{6}$
}

\begin{abstract}
Interventions to improve antiretroviral therapy (ART) access are urgently needed to maximize the multiple benefits from ART. This pilot study examined the effect of a conditional economic incentive on linkage to care and uptake of treatment following ART referral by a mobile health clinic. Between April 2015 and May 2016, 86 individuals $(\geq 18$ years old) referred for ART in a resource-limited setting were randomized (1:1) to a control group or to an incentive: R300 cash ( $\sim 23$, or 3.5 days minimum wage in the domestic worker sector), conditional upon starting ART within 3 months. Outcome data were obtained from clinic records. The incentive effects on linkage to care (first clinic visit within 3 months) and ART initiation (treatment uptake within 3 months) were assessed using logistic regression. Overall, $67 \%$ linked to care and $42 \%$ initiated ART within 3 months after referral. No significant differences were found between the incentive and non-incentive group in terms of linkage to care [adjusted odds ratio (aOR): 0.70 , 95\% confidence interval (CI): 0.26-1.91] and initiation of ART (aOR: 0.67, 95\% CI: 0.26-1.78). Ordinary leastsquares regression analysis showed that incentivized individuals linked to care in fewer days $(-7.9,95 \%$ CI: -18.09 to 2.26) and started treatment in fewer days (-7.3, 95\% CI: -27.01 to 12.38$)$, but neither result was statistically significant. Our findings demonstrate poor treatment uptake by both the intervention and control participants and further highlight the challenge in achieving universal early treatment access. Further research is required to understand how economic incentives, which have been shown to have many benefits, can be applied to improve linkage to HIV care and treatment.
\end{abstract}

Keywords: linkage to HIV care, ART initiation, HIV treatment cascade, behavioral economics, economic incentives, Southern Africa

\section{Introduction}

A NTIRETROVIRAL THERAPY (ART) for HIV is key to curbing the effects of the epidemic and essential to achieve an AIDS-free generation. ${ }^{1}$ ART reduces AIDSrelated morbidity and mortality, and people living with HIV can have life expectancy similar to those not infected with HIV if they start treatment early in disease progression. ${ }^{2-6}$ ART-induced viral suppression nearly eliminates the onward transmission of HIV, ${ }^{7,8}$ and therefore, early ART initiation also maximizes the efficacy of ART for HIV prevention. In light of the benefits of early ART initiation, the World Health Organization (WHO) recommends a "treat-all" strategy for ART - all people living with HIV should be eligible for ART regardless of CD4 count-and most countries have adopted this approach. ${ }^{9}$ One of the largest global public health challenges now is to ensure early treatment access for all, ${ }^{10}$ as many patients only seek care when their CD4 count is low and they are experiencing symptoms. ${ }^{11,12}$

South Africa has the largest global epidemic ${ }^{13}$ and adopted a treat-all policy in September, 2016. ${ }^{14}$ Despite having the largest ART program, $\sim 35 \%$ of all people living with HIV in

\footnotetext{
${ }^{1}$ Southern Africa Labour and Development Research Unit (SALDRU), University of Cape Town, Cape Town, South Africa.

${ }^{2}$ The Desmond Tutu HIV Centre, University of Cape Town, Cape Town, South Africa.

${ }^{3}$ Department of Behavioral and Social Sciences, Brown University School of Public Health, Providence, Rhode Island.

${ }^{4}$ Department of Psychiatry and Mental Health, University of Cape Town, Cape Town, South Africa.

${ }^{5}$ Department of Epidemiology, Brown University School of Public Health, Providence, Rhode Island.

${ }^{6}$ Department of Health Services, Policy and Practice (HSPP), Brown University School of Public Health, Providence, Rhode Island.
} 
the country are not on treatment. ${ }^{14,15}$ Studies demonstrate that between $32 \%$ and $50 \%$ of patients referred for ART at public sector hospitals and clinics do not initiate treatment by study end-line, which ranged from 3 to 24 months. ${ }^{16-19}$ Moreover, the proportion of patients linked to care following community-based HIV testing (e.g., at mobile clinics) has been particularly low. ${ }^{20,21}$ Community-based services are effective at reaching previously undiagnosed HIVpositive individuals ${ }^{22}$ and can be an effective component of the treat-all strategy if we improve linkage to care from these services.

Behavioral economic theory sheds light on why individuals may delay ART initiation. Within a behavioral economic framework, decision-making is not viewed as strictly rational, as outlined in traditional microeconomic theory, and costbenefit calculations are strongly influenced by contextual and other psychosocial factors. ${ }^{23}$ Multiple barriers to treatment access have been documented, indicating a high potential cost to ART uptake. Structural barriers include lack of money for food or transport to clinics and lack of time. ${ }^{24}$ Psychosocial factors include fear of being stigmatized and fear of treatment side effects. ${ }^{25-27}$ In South Africa, individual impediments to early ART access have historically been compounded by the requirement of patients to make multiple, and time-consuming, clinic visits to initiate ART, with patients typically making 4-6 visits before receiving medication. ${ }^{28,29}$ The process included HIV diagnosis, ART eligibility screening, baseline blood tests and physical examinations, and extensive pre-ART counseling that aimed to improve long-term adherence. ${ }^{30,31}$ The high costs associated with ART initiation help explain why some individuals perceive ART as more of an immediate burden than a benefit and consequently delay treatment.

In addition, for many people, and especially for asymptomatic individuals, the benefits of ART will be perceived to accrue only in the future. The tendency of people to favor immediate rewards and heavily discount future outcomes, often referred to as "temporal discounting,",23 could therefore undermine appreciation of the immediate value of ART and contribute to the decision to delay treatment. Novel interventions, especially within community-based contexts, are needed to reduce the real and perceived costs and/or increase the benefits of early access, and to encourage ART initiation.

Conditional economic incentives (CEIs) - financial (or other) rewards given to individuals who accomplish specific tasks that can be objectively monitored ${ }^{32}$ - have potential to improve ART uptake. Theoretically, an incentive, especially a financial incentive, could decrease the actual costs of starting treatment (e.g., transport costs) and have a price effect on demand. A CEI could also increase the value of ART initiation, especially for asymptomatic patients, by providing a near-immediate benefit. A CEI therefore has the potential both to reduce the costs and increase the benefits of ART and to alter the cost-benefit analysis of decision making. In practice, CEIs have been demonstrated to improve a range of other HIV prevention and treatment outcomes, including adherence to ART, ${ }^{32}$ HIV testing, ${ }^{33}$ uptake of and retention on prevention of mother-to-child transmission (PMTCT) services, ${ }^{34}$ uptake of voluntary medical male circumcision, ${ }^{35}$ reduction in risky sexual behavior, ${ }^{36,37}$ and reduced HIV infections in adolescent girls. ${ }^{38}$

While this is an area of growing research interest, there are few published studies that assess the impact of CEIs on linkage to HIV care and ART initiation. ${ }^{39}$ There is a paucity of data on the effect of a cash incentive on ART initiation. One study tested an intervention that provided injection drug users in India the opportunity to earn up to 15 vouchers (redeemable for groceries or household items): the first for ART initiation ( $\$ 4)$, 12 for follow-up visits ( $\$ 4$ each), and 2 for viral suppression (\$8 each). Results showed that incentivized individuals were significantly more likely to initiate ART. ${ }^{40}$ In the United States, however, incentives (\$25 and \$100 gift cards for the receipt of medical care) did not improve the proportions who linked to care within 3 months. ${ }^{41}$ The effect of an incentive on decision making will be strongly influenced variously by the incentive approach, the context, and the specific population. As such, more research is needed on different incentives in different contexts to better understand how to maximize the efficacy of incentives for treatment uptake. ${ }^{39}$

This study examined the effect of a CEI (R300/\$23 cash for ART initiation within 3 months) on linkage to care and uptake of treatment among adult men and women referred for ART by a mobile health clinic in Cape Town, South Africa.

\section{Materials and Methods}

\section{Study setting and sample}

From April 2015 to May 2016, the iLink Study enrolled individuals diagnosed HIV positive and referred for ART by a mobile health clinic in Cape Town. ART referral was based on National Department of Health guidelines, at that time: a CD4 count $\leq 500$ cells $/ \mu \mathrm{L}^{42}$ The mobile clinic is operated by an organization well known to local communities and has been offering free screening for several health conditions in resource-poor areas since 2008 . The clinic frequently returns to several locations within the study site, an informal urban area consisting mainly of "shack" type houses built from corrugated metal sheets nailed to timber frames. Locations are typically areas with high foot traffic, such as at the corner of a large intersection or adjacent to large public transport terminals. Clinic services are provided for several hours each day at each location from within counseling rooms in the vehicle and its trailer, and in tents erected alongside the vehicle. Services are advertised with banners and through the distribution of pamphlets to people passing by and residents in the area.

Patients assessed as ART eligible at the mobile clinic are provided counseling on the benefits of early ART and referral to ART services. For the iLink Study, individuals were screened for study eligibility by the mobile clinic staff immediately after referral for ART. Study eligibility criteria included being 18 years or older, never having been on ART, and owning a cell phone. The Human Research Ethics Committee, University of Cape Town, provided study approval (ref: 849/2014). Written informed consent was provided by all participants. The iLink Study is registered at clinicaltrials.gov (NCT02440386).

For this pilot study, a sample size of 84 participants (42 in the control group and 42 in the intervention group) was estimated to provide $80 \%$ power to detect a 30 -percentage point difference in ART initiation by month 3 (alpha=0.05), assuming that $40 \%$ of individuals in the control group would start treatment within 3 months.

\section{Intervention}

Eligible participants completed a baseline survey and were randomly assigned (1:1) to the control or intervention 
group. Baseline fieldwork was conducted by the mobile clinic's counselors and nurses. All participants received a R50 ( \$4) grocery voucher as a token of appreciation for study enrolment. The control group received the standard of care: follow-up telephone counseling by the mobile clinic staff to encourage linkage to care. The intervention group received the standard of care plus a voucher that could be exchanged for R300 cash ( \$23) if ART was started within 3 months. In 2015, R300 in the City of Cape Town was equivalent to $\sim 3.5$ days wage at minimum wage for workers within the Domestic Worker Sector. ${ }^{43}$ According to the 2014/2015 Living Conditions Survey, R300 was also equivalent to approximately a third of the average monthly expenditure on food, beverages, and tobacco for households in informal urban areas. ${ }^{44}$ As the mobile health clinic targets the poorest populations of informal urban areas in Cape Town, the incentive would almost certainly have been equivalent to considerably more than a third of participants' monthly household expenditure on food.

Participants assigned to the intervention received a small card in their home language, with the expiry date (i.e., 3 months from study enrolment) on the front of the card. The back of the card provided participants with a reminder of what they needed to do to receive the incentive: (1) contact the study team (using a free and well-known "please call me" mobile service) after initiating ART, and (2) schedule a meeting with a fieldworker to verify ART initiation by presenting their first batch of ART medicines (for images of the card, see Supplementary Data 1 at www.liebertpub.com/apc). The mobile clinic staff carefully explained the incentive system to participants and provided participants the opportunity to ask clarifying questions. Throughout the baseline fieldwork, the clinic staff used language, such as "we" and "our study," to brand the intervention as a collaboration between the University research team and the mobile clinic.

Verification of ART initiation and delivery of the incentive were conducted by the University-affiliated research team at a mutually agreed-upon location convenient and safe for both participants and fieldworkers. In practice, these meetings generally occurred within 5 min walking distance from participants' homes, at locations such as petrol stations, corner stores, or shopping centers.

\section{Data}

At baseline, a face-to-face survey was conducted to collect demographic and socioeconomic data, as well as data on factors that could influence ART initiation. Outcome data on linkage to care and ART initiation were collected from three sources. First, during verification of ART among intervention participants, fieldworkers recorded the date of ART initiation from the participant's ART package and collected data on first clinic visits.

Second, a follow-up telephone survey was conducted at month 3. Extensive efforts were made to reduce attrition, including six contact attempts, with three outside regular working hours, and cell phone messaging to schedule the telephone survey. Data on dates of both first clinic visit and ART initiation were collected.

Third, participants were linked first to clinic folder numbers in the Department of Health's Provincial database and then to deidentified clinic records. We used a four-phase matching approach. First, we identified exact matches on names and dates of birth. Second, we used well-known variants in the spelling of names and matched to correct dates of birth. Third, we allowed the day of birth to vary by two digits. Fourth, for verification of matching, we provided to the Province a list of study participants for whom the match to a clinic record was uncertain. Data were available on dates of clinic visits, dates of CD4 count laboratory tests, and the dates on which ART was dispensed. We assessed the completeness of matching using clinic record outcomes for study participants who met with the study team and verified ART initiation. Among these individuals, 94\% (17/18) had initiated ART according to the clinic records, thus suggesting a high degree of accuracy in data matching.

\section{Measures}

"Linkage to care" was defined as visiting a health facility within 3 months from study enrolment. Three months is a common time period used, and a period proposed to standardize measures, for linkage to care and treatment studies. ${ }^{45}$ Our main measures of linkage to care and ART initiation were based on the clinic record data. These data are preferred to the survey data as they are not influenced by survey attrition or self-reporting bias. In one case, clinic visit data were missing, but we assumed linkage to care as the laboratory data indicated that a CD4 count test had been conducted 15 days after study enrolment. Individuals without any record of clinic attendance were assumed not to have linked to care.

ART initiation was defined as receipt of ART within 3 months. We used the date on which ART was first dispensed to identify ART initiation. In one case, no data on drug dispensing were available and we assigned the individual an ART start date based on an "ART visit," as recorded in the clinic records. This is used to identify ART-related services from the day of initiation, and the first such entry would indicate the date of initiation.

\section{Analysis}

We first present baseline sample characteristics by assignment to study group to assess whether there were any differences in key characteristics following randomization. Key characteristics included a range of demographic, socioeconomic, and psychosocial factors associated with linkage to care or ART initiation. ${ }^{24}$

We assessed the incentive effect on linkage to care and ART initiation using bivariate and multivariable logistic regression models. To increase the precision of estimates in the multivariable analysis, we controlled for key demographic characteristics (age, gender, and education) and all other factors that could influence ART initiation, which were significantly different $(p<0.1)$ across study groups at baseline. To assess whether the incentive influenced how quickly individuals linked to care or started treatment, we compared the average number of days to linkage to care and ART initiation using multivariable ordinary least-squares (OLS) regression models.

As a sensitivity analysis, we repeated all analyses using equivalent outcome measures created based on the survey data, rather than the clinic record data. While our assessment of the clinic record data indicated a good match between 
study participants and their clinic records, it is still possible that incomplete matching may have biased results. All analyses were conducted using Stata 14.0 (Stata Corporation LP, College Station, TX).

\section{Results}

The study profile is presented in Fig. 1. Of the 112/147 (76\%) participants eligible for enrolment, 87 (78\%) enrolled in the study. The main reason for being screened as ineligible for the study was not owning a cell phone $(n=10)$. Eighteen percent of eligible participants refused to participate. One individual was excluded from the study after enrolment for not meeting a study eligibility criterion. Forty-six (53\%) participants were assigned to the intervention group and all received the intervention. Our main analysis includes all 86 study participants as it is assumed that clinic record data are complete.

Table 1 presents baseline sample characteristics by study group. Overall, the sample comprised mainly black African individuals (97\%) and women (64\%), with an average age of 33. Most participants $(52 \%)$ estimated that their walk from home to the nearest health clinic would take less than $30 \mathrm{~min}$, and $36 \%$ lived at a 30- to 60-min walk from the nearest clinic. The average of participants' estimates of the cost for public transport from their home to the closest health clinic was R10.33. Self-assessed depression was common and only half the sample reported good health. Most participants $(88 \%)$ reported having had an HIV test before and a significant proportion (37\%) did not think it was at all likely that they would test HIV positive on the day of study enrolment. While many participants reported internalized and perceived stigma, and the belief that they would experience ART side effects, the vast majority were very ready and motivated for ART.

Gender was the only demographic factor with a relatively large difference between study groups at baseline (intervention group: $44 \%$ male; control group: $28 \%$ male; $p=0.12$ ). There was no significant difference in health between groups. In terms of psychosocial factors, individuals in the control group were more likely to have witnessed a positive ART effect, that is, they knew a friend or family member taking ART and believed ART had a positive health effect (75\% vs. $57 \% ; p=0.07)$. Control group participants were also more likely to report perceived stigma - the perception that they would be treated unfairly or badly by their spouse, family, friends, a member of their community, or a health professional $(82 \%$ vs. $53 \% ; p<0.01)$.

\section{Intervention effect on linkage to care and $A R T$ initiation}

Among the full sample, 67\% $(n=58,95 \%$ CI: 57-78) linked to care within 3 months. Participants in the intervention group were less likely to link to care, but the result was not statistically significant (Table 2, columns 1-3; aOR: 0.70; 95\% CI: $0.26-1.91 ; p=0.49)$. Sensitivity analysis using outcome measures based on survey data (see Supplementary Table S1, Supplementary Data 2) found almost no difference in linkage to care across study groups (aOR: 1.07; 95\% CI: $0.16-6.92 ; p=0.95)$.

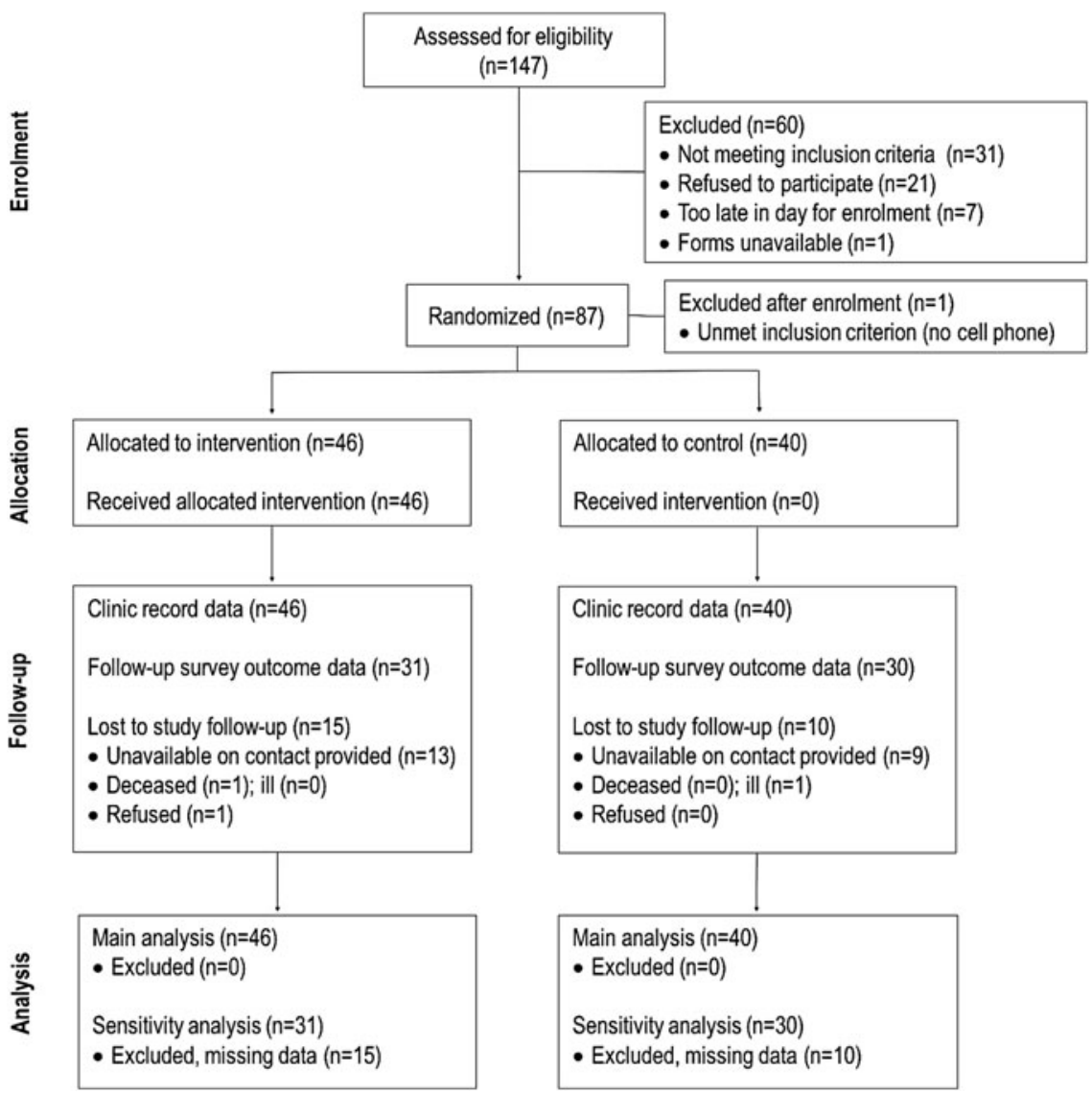


Table 1. Baseline Sample Characteristics By Study Group

\begin{tabular}{|c|c|c|c|c|c|c|c|c|}
\hline & \multicolumn{2}{|c|}{ Full sample } & \multicolumn{2}{|c|}{ Intervention } & \multicolumn{2}{|r|}{ Control } & \multirow{2}{*}{$\begin{array}{c}\text { Difference } \\
\text { (intervention } \\
\text { vs. control) }\end{array}$} & \multirow[b]{2}{*}{$\mathrm{p}$} \\
\hline & $\mathrm{N}$ & $\%$ or mean ${ }^{\mathrm{a}}$ & $\mathrm{n}$ & $\%$ or mean ${ }^{\mathrm{a}}$ & $\mathrm{n}$ & $\%$ or mean ${ }^{\mathrm{a}}$ & & \\
\hline \multicolumn{9}{|l|}{ Demographics/SES } \\
\hline Male & 86 & 36.0 & 46 & 43.5 & 40 & 27.5 & 16.0 & 0.12 \\
\hline Age in years (mean) & 86 & 33.0 & 46 & 32.5 & 40 & 33.7 & -1.2 & 0.54 \\
\hline Black African & 86 & 96.5 & 46 & 95.7 & 40 & 97.5 & -1.8 & 0.64 \\
\hline Married & 86 & 20.9 & 46 & 19.6 & 40 & 22.5 & -2.9 & 0.74 \\
\hline Years of education (mean) & 86 & 10.0 & 46 & 10.1 & 40 & 9.9 & 0.2 & 0.64 \\
\hline Employed & 86 & 32.6 & 46 & 32.6 & 40 & 32.5 & 0.1 & 0.99 \\
\hline Dwelling type: shack & 86 & 76.7 & 46 & 80.4 & 40 & 72.5 & 7.9 & 0.39 \\
\hline Food insecurity & 85 & 58.8 & 45 & 57.8 & 40 & 60.0 & -2.2 & 0.84 \\
\hline Time to walk from home to clinic & 84 & & 44 & & 40 & & & \\
\hline$<30 \mathrm{~min}$ & & 52.4 & & 54.5 & & 50.0 & 4.5 & 0.68 \\
\hline $30-60 \mathrm{~min}$ & & 35.7 & & 34.1 & & 37.5 & -3.4 & 0.75 \\
\hline$>60 \mathrm{~min}$ & & 11.9 & & 11.4 & & 12.5 & -1.1 & 0.87 \\
\hline $\begin{array}{l}\text { Cost of public transport to clinic } \\
\text { (mean of estimate in Rands) }\end{array}$ & 79 & 10.33 & 44 & 10.21 & 35 & 10.48 & -0.27 & 0.80 \\
\hline \multicolumn{9}{|l|}{ Health } \\
\hline CD4 count (mean) & 86 & 313.8 & 46 & 303.3 & 40 & 325.8 & -22.4 & 0.36 \\
\hline Health $=$ good & 86 & 51.2 & 46 & 54.3 & 40 & 47.5 & 6.8 & 0.53 \\
\hline Depressed sometimes & 86 & 72.1 & 46 & 71.7 & 40 & 72.5 & -0.8 & 0.94 \\
\hline No previous HIV test & 86 & 11.6 & 46 & 13.0 & 40 & 10.0 & 3.0 & 0.66 \\
\hline \multicolumn{9}{|l|}{ Psychosocial } \\
\hline Believed unlikely to test HIV+ & 83 & 37.3 & 46 & 39.1 & 37 & 35.1 & 4.0 & 0.71 \\
\hline Very ready for ART & 86 & 83.7 & 46 & 87.0 & 40 & 80.0 & 7.0 & 0.39 \\
\hline Very motivated for ART & 85 & 84.7 & 46 & 87.0 & 39 & 82.1 & 4.9 & 0.54 \\
\hline Very confident of positive ART effect & 86 & 88.4 & 46 & 84.8 & 40 & 92.5 & -7.7 & 0.26 \\
\hline Heard of ART & 86 & 94.2 & 46 & 93.5 & 40 & 95.0 & -1.5 & 0.76 \\
\hline Knowledge of ART $=$ good & 86 & 79.1 & 46 & 78.3 & 40 & 80.0 & -1.7 & 0.84 \\
\hline ART is not a cure & 86 & 87.2 & 46 & 89.1 & 40 & 85.0 & 4.1 & 0.57 \\
\hline Likely to experience side effects & 85 & 35.3 & 46 & 28.3 & 39 & 43.6 & -15.3 & 0.15 \\
\hline Know someone who died of AIDS & 85 & 68.2 & 46 & 71.7 & 39 & 64.1 & 7.6 & 0.46 \\
\hline Know someone on ART & 86 & 68.6 & 46 & 63.0 & 40 & 75.0 & -12.0 & 0.23 \\
\hline Witnessed positive ART effect & 86 & 65.1 & 46 & 56.5 & 40 & 75.0 & -18.5 & 0.07 \\
\hline Feels guilty about HIV & 85 & 50.6 & 46 & 43.5 & 39 & 59.0 & -15.5 & 0.16 \\
\hline Feels ashamed to have HIV & 85 & 37.6 & 46 & 34.8 & 39 & 41.0 & -6.2 & 0.56 \\
\hline Perceived stigma (any) & 84 & 66.7 & 45 & 53.3 & 39 & 82.1 & -28.7 & 0.00 \\
\hline Very likely to disclose to partner & 70 & 81.4 & 39 & 87.2 & 31 & 74.2 & 13.0 & 0.18 \\
\hline
\end{tabular}

$p$ Values are for statistical difference tests ( $t$-test for linear variables and Chi-squared test for categorical variables) of the difference between the intervention and control group.

${ }^{a}$ All figures in the column represent percentages, unless otherwise stated.

ART, antiretroviral therapy; SES, socioeconomic status.

Among the full sample, $42 \%(n=36,95 \%$ CI: $31-52)$ initiated ART within 3 months. Table 2 (column 4) indicates that a greater percentage of individuals in the control group initiated ART. Similarly, multivariable regression analysis (column 6) showed that individuals in the treatment group were less likely to initiate ART, but the difference was not statistically significant (aOR: $0.67 ; 95 \%$ CI: $0.26-1.61 ; p=0.42$ ). Sensitivity analysis using outcome measures based on survey data (see Supplementary Table S1, Supplementary Data 2) found almost no difference in ART initiation across study groups (aOR: 1.05; 95\% CI: 0.30-3.70; $p=0.94$ ).

Among participants who linked to care within 3 months, the average number of days to linkage to care was lower in the intervention group (11.5 vs. 17 days). Multivariable OLS regression analysis (Table 3, Model 1) found individuals in the intervention group to visit a clinic $\sim 8$ days quicker on average $(-7.9 ; 95 \% \mathrm{CI}:-18.1$ to $2.3 ; p=0.13)$. Similarly, among participants who did initiate treatment (Model 2), the average number of days to treatment uptake was lower among the intervention group ( 24.5 vs. 31 days; $\beta$ : $-7.3 ; 95 \% \mathrm{CI}:-27$ to $12 ; p=0.45)$. Sensitivity analyses using outcome measures based on survey data (see Supplementary Table S2, Supplementary Data 2) found larger treatment effects on both days to linkage to care $(\beta:-19.1 ; 95 \% \mathrm{CI}:-33.4$ to $-4.8 ; p<0.01)$ and days to treatment uptake $(\beta$ : $-16.6 ; 95 \% \mathrm{CI}:-36.5$ to 3.3 ; $p=0.099$ ).

\section{Discussion}

The therapeutic and prevention gains from ART can be large, but are only fully realized if individuals initiate treatment before their CD4 count drops too low. Our results indicate that the efficacy of ART continues to be undermined with poor rates of ART uptake after referral for treatment by 
Table 2. Intervention Effect on Linkage to Care and Antiretroviral Therapy Initiation

\begin{tabular}{|c|c|c|c|c|c|c|}
\hline & \multicolumn{3}{|c|}{$\begin{array}{l}\text { Linkage to care } \\
\text { within } 3 \text { months }\end{array}$} & \multicolumn{3}{|c|}{$\begin{array}{l}\text { ART initiation } \\
\text { within } 3 \text { months }\end{array}$} \\
\hline & 1 & 2 & 3 & 4 & 5 & 6 \\
\hline & $\begin{array}{c}\% \\
{[95 \% \text { CI] }}\end{array}$ & $\begin{array}{c}O R \\
{[95 \% C I]}\end{array}$ & $\begin{array}{c}a O R \\
{[95 \% C I]}\end{array}$ & $\begin{array}{c}\% \\
{[95 \% \text { CI] }}\end{array}$ & $\begin{array}{c}O R \\
{[95 \% \mathrm{CI}]}\end{array}$ & $\begin{array}{c}a O R \\
{[95 \% C I]}\end{array}$ \\
\hline $\begin{array}{l}\text { Intervention received } \\
\text { No } \\
\text { Yes }\end{array}$ & $\begin{array}{l}72.5[59-86] \\
63[49-77]\end{array}$ & $\begin{array}{c}\operatorname{Ref} \\
0.65[0.26-1.61]\end{array}$ & $\begin{array}{c}\operatorname{Ref} \\
0.70[0.26-1.91]\end{array}$ & $\begin{array}{l}45[30-60] \\
39[25-53]\end{array}$ & $\begin{array}{c}\operatorname{Ref} \\
0.79[0.33-1.89]\end{array}$ & $\begin{array}{c}\operatorname{Ref} \\
0.67[0.26-1.78]\end{array}$ \\
\hline $\begin{array}{l}\text { Control variables } \\
\text { Male (ref: female) } \\
\text { Age (years) } \\
\text { Education (years) } \\
\text { Witnessed ART } \\
\text { effect (ref: no) } \\
\text { Perceived stigma }\end{array}$ & NA & NA & $\begin{array}{l}1.06[0.38-2.94] \\
1.03[0.97-1.10] \\
1.04[0.83-1.30] \\
1.66[0.61-4.48]\end{array}$ & NA & NA & $\begin{array}{l}0.92[0.34-2.49] \\
1.01[0.96-1.07] \\
0.92[0.75-1.14] \\
1.57[0.57-4.27]\end{array}$ \\
\hline $\begin{array}{l}\text { (ret: none) } \\
\text { Observations }\end{array}$ & 86 & 86 & 84 & 86 & 86 & 84 \\
\hline
\end{tabular}

$* * * p<0.01, * * p<0.05, * p<0.1$.

95\% CI, 95\% confidence interval; aOR, adjusted odds ratios; ART, antiretroviral therapy; NA, not applicable; OR, odds ratio; Ref, the reference category.

mobile health clinics. Our findings are consistent with other studies in South Africa that demonstrate poor linkage to care and treatment uptake, ${ }^{16,18,20,46,47}$ and further highlight the challenge in achieving universal early treatment access.

A conditional cash incentive was not found to improve the proportion of patients who linked to care or started treatment within 3 months. Results indicate that incentivized individuals may have sought out care and treatment more quickly (by approximately a week), but these findings were not statistically significant.

The key question is why a cash incentive would not change outcomes among a population that was very poor and experiencing food insecurity. One possibility is that our cash incentive was not perceived to be of value. This is highly unlikely given that our incentive was equivalent to approximately a third of the average monthly household expenditure on food, beverages and tobacco in informal urban areas in South Africa in 2014/2015. ${ }^{44}$ Further, a study conducted among unemployed men in Cape Town between 2008 and 2010 found that an R80 incentive increased demand for HIV testing services. ${ }^{48}$ Their findings demonstrate that an incentive of just over a third of the value of the one used in our study-after adjusting for inflation to compare values in 2015 - was enough to motivate behavior change within a comparable study context.

Follow-up in-depth qualitative interviews $(N=41)$ conducted as part of the iLink Study provided some insights into why our incentive may not have been effective. A recently published article using data from the qualitative substudy revealed large and multiple barriers to treatment uptake, including an array of clinic-related barriers that made it difficult for patients to start treatment and that compounded other

Table 3. Intervention Effect on Average Number of Days to Linkage to CARE AND ANTIRETroviral Therapy Initiation

\begin{tabular}{|c|c|c|}
\hline & Days to linkage & Days to $A R T$ \\
\hline & 1 & 2 \\
\hline & $O L S[95 \% C I]$ & $O L S[95 \% C I]$ \\
\hline \multicolumn{3}{|l|}{ Intervention received } \\
\hline No & Ref & Ref \\
\hline Yes & $-7.92[-18.09$ to 2.26$]$ & $-7.32[-27.01$ to 12.38$]$ \\
\hline \multicolumn{3}{|l|}{ Control variables } \\
\hline Male (ref: female) & $-3.71[-14.08$ to 6.65$]$ & $-15.08[-35.75$ to 5.59$]$ \\
\hline Age (years) & $0.05[-0.59$ to 0.70$]$ & $0.04[-1.10$ to 1.18$]$ \\
\hline Education (years) & $1.66[-0.67$ to 4.00$]$ & $-0.60[-4.73$ to 3.53$]$ \\
\hline Witnessed ART effect (ref: no) & $-12.03 * *[-22.98$ to -1.08$]$ & $-16.27 *[-35.54$ to 3.00$]$ \\
\hline Perceived stigma (ref: none) & $-4.80[-15.31$ to 5.70$]$ & $-9.01[-26.85$ to 8.84$]$ \\
\hline Observations & 56 & 34 \\
\hline$r$-squared & 0.16 & 0.19 \\
\hline
\end{tabular}

$* * * p<0.01, * * p<0.05, * p<0.1$.

95\% CI, 95\% confidence interval; ART, antiretroviral therapy; OLS, ordinary least-squares regression coefficients; Ref, the reference category. 
psychosocial and structural barriers. Study participants typically had to make 4-6 clinic visits before ART initiation, and several participants in the incentive group reported giving up on the process. ${ }^{28}$ These qualitative findings raise the possibility that the incentive amount was not large enough to overcome the high costs associated with ART initiation. In other words, the incentive may have been too small to provide adequate motivation for participants to complete the onerous ART initiation process.

Given the study context, the approach of using a conditional incentive may also have undermined the intervention. An incentive involving a future payment may only be effective in countering immediate structural barriers, for example, transport money to clinics, if it enables individuals to borrow money in the present to cover related costs. As social capital and the availability of funds within networks are limited in exceptionally resource-poor settings, participants may not have been able to borrow against the future and therefore the intervention could have been ineffective in reducing costs to treatment uptake.

In addition, an incentive involving a future payment may only be effective in altering the perceived costs and benefits associated with ART uptake if people trust that the incentive will be paid to them. Further analysis of the iLink Study's indepth interviews $(N=41)$ was conducted for this study to examine participant's perceptions of the incentive. Several individuals in the intervention group expressed either uncertainty about whether they would actually receive the incentive or not, or complete distrust in the incentive. Several aspects of our intervention were designed with the aim of instilling trust in the incentive. The voucher given to respondents was printed in color on high-quality paper and included the University logo. Moreover, the R50 shopping voucher given as a token of appreciation for study participation was intended to signal that the study would deliver the incentive. These efforts appear not to have been completely successful in overcoming the low levels of general trust found among people in the region. ${ }^{49}$

The conditionality of the incentive could potentially have also limited the desired effect of increasing the perceived benefits of ART uptake. As people have a tendency to discount the value of future benefits, it was hoped that the incentive would increase the present value of ART initiation, especially among asymptomatic patients. In our sample of extremely poor individuals who would likely have been focused on immediate needs rather than future benefits, it is possible that the value of our incentive was also subject to temporal discounting even though the time frame (maximum of 3 months) was relatively short.

The study context and design point toward another possible explanation for the lack of an incentive effect. The incentive was one of several factors that may have helped motivate linkage to care and treatment services. First, the standard of care at the mobile health clinic involves intensive follow-up telephone calls to encourage linkage. Second, the R50 shopping voucher provided at enrolment as a token of appreciation was reported by some participants to have helped with linkage to care as it enabled purchasing food to be taken with their ART. Third, the baseline study processes created additional time for participants to spend with counselors. If any, or a combination of any, of these factors was as effective in "nudging" participants uncertain about starting treatment as the incentive was, then that would have undermined an incentive effect. It is thus possible that the incentive might have better value among populations with less linkage to care support.

Our final explanation for the study findings is that the small sample size, which is a study limitation, may simply have resulted in a null result due to small sample size bias. Given the small sample size, and the study context, the degree to which the findings apply to other samples and populations is unknown.

Our study findings have several implications for future research and policy. First, the pilot study has demonstrated that all aspects of the study design are feasible within the study setting and could be replicated to test other linkage to care interventions. Second, further research on different incentive approaches within this setting is required. For example, multiple small incentives for completion of every step of the ART initiation process, at shorter intervals, may help to overcome issues of trust and discounting that may have undermined our incentive; may provide more immediate positive reenforcement; and may help to counter structural barriers (e.g., the transport costs of subsequent clinic visits). Third, the finding that a financial incentive did not affect treatment outcomes among a population living in poverty indicates that barriers to ART were substantial. Research to identify and reduce these barriers is important, as interventions designed to encourage individuals to start treatment through gentle nudges may be ineffectual while barriers remain high. For example, our finding that a substantial proportion (over a third) of participants linked to care, but did not start treatment within 3 months, aligns with qualitative evidence indicating significant clinicrelated barriers to ART initiation. ${ }^{28}$ These findings suggest that models of ART initiation that are patient centered, such as same-day ART initiation and models with more efficient ART preparation, ${ }^{29,50,51}$ may improve ART uptake.

In conclusion, the use of a once-off cash incentive conditional upon ART initiation was not found to improve treatment uptake in our setting. Further research is required to understand how economic incentives, which have been shown to have many benefits where other dimensions of HIV/ AIDS are concerned, can be applied to improve linkage to HIV care and treatment.

\section{Acknowledgments}

The authors gratefully acknowledge the staff of the Tutu Tester Mobile Clinic for their valuable assistance with developing the study materials and with data collection. We are also grateful for data access from the Western Cape Government Department of Health and assistance in linking study participants to electronic clinic records from Andrew Boulle, Meg Osler, Jonathan Euvrard, and Nicki Tiffin (Centre for Infectious Disease Epidemiology and Research, University of Cape Town, and the Provincial Health Data Centre, Western Cape Government).

Source of funding: this study was partially funded by the National Research Foundation, South Africa, through the Research Career Advancement Fellowship. Support for this study was also provided by the South African Social Science and HIV (SASH) Programme, an initiative funded by the Eunice Kennedy Shriver National Institute of Child Health and Human Development of the National Institutes of Health 
(Award No. R24HD077976). C.K. and M.N.L. derived support for analysis, interpretation, and writing from the National Institute of Mental Health (grants K01MH 096646 and R01 MH106600). The content is solely the responsibility of the authors and does not necessarily represent the official views of the funders.

\section{Author Disclosure Statement}

No competing financial interests exist.

\section{References}

1. Fauci AS, Folkers GK. Toward an AIDS-free generation. JAMA 2012;308:343-344.

2. Bor J, Herbst AJ, Newell M-L, et al. Increases in adult life expectancy in rural South Africa: Valuing the scale-up of HIV treatment. Science 2013;339:961-965.

3. The TEMPRANO ANRS 12136 Study Group. A trial of early antiretrovirals and isoniazid preventive therapy in Africa. N Engl J Med 2015;373:808-822.

4. The INSIGHT START Study Group. Initiation of antiretroviral therapy in early asymptomatic HIV infection. $\mathrm{N}$ Engl J Med 2015;373:795-807.

5. Johnson LF, Mossong J, Dorrington RE, et al. Life expectancies of South African adults starting antiretroviral treatment: Collaborative analysis of cohort studies. PLoS Med 2013;10:e1001418.

6. Trickey A, May MT, Vehreschild J-J, et al. Survival of HIV-positive patients starting antiretroviral therapy between 1996 and 2013: A collaborative analysis of cohort studies. Lancet HIV 2017;4:e346-e356.

7. Cohen MS, Chen YQ, McCauley M, et al. Prevention of HIV-1 infection with early antiretroviral therapy. N Engl J Med 2011;365:493-505.

8. Tanser F, Bärnighausen T, Grapsa E, et al. High coverage of ART associated with decline in risk of HIV acquisition in rural KwaZulu-Natal, South Africa. Science 2013;339: 966-971.

9. WHO. Consolidated Guidelines on the Use of Antiretroviral Drugs for Treating and Preventing HIV Infection. Geneva: WHO, 2016.

10. Katz IT, Maughan-Brown B. Improved life expectancy of people living with HIV: Who is left behind? Lancet HIV 2017;4:e324-e326.

11. Siedner MJ, Ng CK, Bassett IV, et al. Trends in CD4 count at presentation to care and treatment initiation in subSaharan Africa, 2002-2013: A meta-analysis. Clin Infect Dis 2015;60:1120-1127.

12. Lahuerta M, Wu Y, Hoffman S, et al. Advanced HIV disease at entry into HIV care and initiation of antiretroviral therapy during 2006-2011: Findings from four sub-Saharan African countries. Clin Infect Dis 2014;58:432-441.

13. UNAIDS. Global AIDS Update 2016. Geneva: Joint United Nations Programme on HIV/AIDS (UNAIDS), 2016.

14. Motsoaledi A. Health Dept Budget Vote Speech 2016/17. Department of Health, South Africa; 2016. Available at: www .gov.za/speeches/debate-health-budget-vote-national-assembly10-may-2016-dr-aaron-motsoaledi-minister-health (Last accessed January 19, 2018).

15. UNAIDS. UNAIDS Data 2017. Geneva: Joint United Nations Programme on HIV/AIDS (UNAIDS), 2017.

16. Kranzer K, Zeinecker J, Ginsberg P, et al. Linkage to HIV care and antiretroviral therapy in Cape Town, South Africa. PLoS One 2010;5:e13801.
17. Ingle SM, May $\mathbf{M}$, Uebel $\mathrm{K}$, et al. Outcomes in patients waiting for antiretroviral treatment in the Free State Province, South Africa: Prospective linkage study. AIDS 2010; 24:2717-2725.

18. Patten GE, Wilkinson L, Conradie K, et al. Impact on ART initiation of point-of-care CD4 testing at HIV diagnosis among HIV-positive youth in Khayelitsha, South Africa. J Int AIDS Soc 2013;16:18518.

19. Larson BA, Schnippel K, Brennan A, et al. Same-day CD4 testing to improve uptake of HIV care and treatment in South Africa: Point-of-care is not enough. AIDS Res Treat 2013;2013:941493.

20. Govindasamy D, Kranzer K, van Schaik N, et al. Linkage to HIV, TB and non-communicable disease care from a mobile testing unit in Cape Town, South Africa. PLoS One 2013;8:e80017.

21. Iwuji CC, Orne-Gliemann J, Larmarange J, et al. Uptake of home-based HIV testing, linkage to care, and community attitudes about ART in rural KwaZulu-Natal, South Africa: Descriptive results from the first phase of the ANRS 12249 TasP cluster-randomised trial. PLoS Med 2016;13:e1002107.

22. Tabana H, Nkonki L, Hongoro $C$, et al. A costeffectiveness analysis of a home-based HIV counselling and testing intervention versus the standard (facility based) HIV testing strategy in rural South Africa. PLoS One 2015;10:e0135048.

23. Operario D, Kuo C, Sosa-Rubi SG, et al. Conditional economic incentives for reducing HIV risk behaviors: Integration of psychology and behavioral economics. Health Psychol 2013;32:932-940.

24. Govindasamy D, Ford N, Kranzer K. Risk factors, barriers and facilitators for linkage to antiretroviral therapy care: A systematic review. AIDS 2012;26:2059-2067.

25. Fox MP, Mazimba A, Seidenberg P, et al. Barriers to initiation of antiretroviral treatment in rural and urban areas of Zambia: A cross-sectional study of cost, stigma, and perceptions about ART. J Int AIDS Soc 2010;13:8.

26. Lyons A, Moiane L, Demetria E, Veldkamp P, Prasad R. Patient perspectives on reasons for failure to initiate antiretroviral treatment in Mozambique: A call for compassionate counseling. AIDS Patient Care STDs 2016;30:197199.

27. Smith LR, Amico KR, Shuper PA, et al. Information, motivation, and behavioral skills for early pre-ART engagement in HIV care among patients entering clinical care in KwaZuluNatal, South Africa. AIDS Care 2013;25:1485-1490.

28. Maughan-Brown B, Kuo C, Galárraga O, et al. Stumbling blocks at the clinic: Experiences of seeking HIV treatment and care in South Africa. AIDS Behav 2017. DOI:10.1007/ s10461-017-1877-4.

29. Rosen S, Maskew M, Fox MP, et al. Initiating antiretroviral therapy for HIV at a patient's first clinic visit: The RapIT randomized controlled trial. PLoS Med 2016;13:e1002015.

30. Thompson MA, Aberg JA, Cahn P, et al. Antiretroviral treatment of adult HIV infection: 2010 recommendations of the International AIDS Society-USA Panel. JAMA 2010; 304:321-333.

31. Myer L, Zulliger R, Pienaar D. Diversity of patient preparation activities before initiation of antiretroviral therapy in Cape Town, South Africa. Trop Med Int Health 2012;17: 972-977.

32. Galárraga O, Genberg BL, Martin RA, et al. Conditional economic incentives to improve HIV treatment adherence: 
Literature review and theoretical considerations. AIDS Behav 2013;17:2283-2292.

33. Lee R, Cui RR, Muessig KE, et al. Incentivizing HIV/STI Testing: A systematic review of the literature. AIDS Behav 2014;18:905-912.

34. Yotebieng M, Thirumurthy H, Moracco KE, et al. Conditional cash transfers and uptake of and retention in prevention of mother-to-child HIV transmission care: A randomised controlled trial. Lancet HIV 2016;3:e85e93.

35. Thirumurthy H, Masters SH, Rao S, et al. Effect of providing conditional economic compensation on uptake of voluntary medical male circumcision in Kenya. JAMA 2014;312:703-718.

36. de Walque D, Dow WH, Nathan R, et al. Incentivising safe sex: A randomised trial of conditional cash transfers for HIV and sexually transmitted infection prevention in rural Tanzania. BMJ Open 2012;2:e000747.

37. Nyquist M, Corno L, De Walque D, et al. Using Lotteries to Incentivize Safer Sexual Behavior: Evidence From a Randomized Controlled Trial on HIV Prevention. World Bank, 2015. Available at: https://openknowledge.worldbank.org/ bitstream/handle/10986/21654/WPS7215.pdf?sequence $=1$ (Last accessed January 19, 2018).

38. Baird SJ, Garfein RS, McIntosh CT, et al. Effect of a cash transfer programme for schooling on prevalence of HIV and herpes simplex type 2 in Malawi: A cluster randomised trial. Lancet 2012;379:1320-1329.

39. Bassett IV, Wilson D, Taaffe J, et al. Financial incentives to improve progression through the HIV treatment cascade. Curr Opin HIV AIDS 2015;10:451-463.

40. Solomon SS, Srikrishnan AK, Vasudevan CK, et al. Voucher incentives improve linkage to and retention in care among HIV-infected drug users in Chennai, India. Clin Infect Dis 2014;59:589-595.

41. El-Sadr W, Donnell D, Beauchamp G, et al. Financial incentives for linkage to care and viral suppression among HIV-positive patients. JAMA Intern Med 2017;177:10831092.

42. Department of Health, Republic of South Africa. National Consolidated Guidelines for the Prevention of Mother-toChild Transmission of HIV (PMTCT) and the Management of HIV in Children, Adolescents and Adults. Pretoria: Department of Health, 2014.

43. Department of Labour, Republic of South Africa. Government Gazette. No.38254. Republic of South Africa; 2014. Available at: www.labour.gov.za/DOL/downloads/legislation/sectoraldeterminations/basic-conditions-of-employment/Correction\%
20Notice\%20Domestic\%20worker\%202014.pdf (Last accessed January 19, 2018).

44. Statistics South Africa. Living Conditions of Households in South Africa. Pretoria: Statistics South Africa; 2017. Available at: www.statssa.gov.za/publications/P0310/P03 102014.pdf (Last accessed January 19, 2018).

45. Fox MP, Larson B, Rosen S. Defining retention and attrition in pre-antiretroviral HIV care: Proposals based on experience in Africa. Trop Med Int Health 2012;17:12351244.

46. Bassett IV, Regan S, Luthuli P, et al. Linkage to care following community-based mobile HIV testing compared with clinic-based testing in Umlazi Township, Durban, South Africa. HIV Med 2014;15:367-372.

47. Naik R, Doherty T, Jackson D, et al. Linkage to care following a home-based HIV counselling and testing intervention in rural South Africa. J Int AIDS Soc 2015;18: 19843.

48. Nglazi MD, van Schaik N, Kranzer K, et al. An incentivized HIV counseling and testing program targeting hard-toreach unemployed men in Cape Town, South Africa. J Acquir Immune Defic Syndr 2012;59:e28-e34.

49. Lau YK, Ataguba JE. Investigating the relationship between self-rated health and social capital in South Africa: A multilevel panel data analysis. BMC Public Health 2014; 15:266.

50. Pilcher CD, Ospina-Norvell C, Dasgupta A, et al. The effect of same-day observed initiation of antiretroviral therapy on HIV viral load and treatment outcomes in a U.S. public health setting. J Acquir Immune Defic Syndr 2017; 74:44-51.

51. Wilkinson L, Duvivier H, Patten G, et al. Outcomes from the implementation of a counselling model supporting rapid antiretroviral treatment initiation in a primary healthcare clinic in Khayelitsha, South Africa. South Afr J HIV Med 2015;16:367.

Address correspondence to: Brendan Maughan-Brown, PhD Southern Africa Labour and Development Research Unit (SALDRU) University of Cape Town Private Bag X3 Rondebosch Cape Town 7701 South Africa

E-mail: brendan.maughanbrown@gmail.com 